\title{
FIXED POINT PROPERTY FOR UNIVERSAL LATTICE ON SCHATTEN CLASSES
}

\author{
MASATO MIMURA
}

(Communicated by Marius Junge)

\begin{abstract}
The special linear group $G=\mathrm{SL}_{n}\left(\mathbb{Z}\left[x_{1}, \ldots, x_{k}\right]\right)$ ( $n$ at least 3 and $k$ finite) is called the universal lattice. Let $n$ be at least 4 , and $p$ be any real number in $(1, \infty)$. The main result is the following: any finite index subgroup of $G$ has the fixed point property with respect to every affine isometric action on the space of $p$-Schatten class operators. It is in addition shown that higher rank lattices have the same property. These results are a generalization of previous theorems respectively of the author and of Bader-Furman-GelanderMonod, which treated a commutative $L^{p}$-setting.
\end{abstract}

\section{INTRODUCTION AND MAIN RESULTS}

In this paper, every group is assumed to be locally compact and second countable, and every subgroup of a (topological) group is assumed to be closed. The symbol $k$ is used for representing any finite natural (positive) number, and we set $A=$ $\mathbb{Z}\left[x_{1}, \ldots, x_{k}\right]$. Hereafter, by higher rank lattices we mean lattices (namely, discrete subgroups with finite covolume) in semisimple algebraic groups over local fields (possibly Archimedean) with each factor having local rank $\geq 2$. We use the symbol $\langle\cdot \mid \cdot\rangle$ for the inner product on a Hilbert space.

The special linear group $G=\mathrm{SL}_{n}(A)=\mathrm{SL}_{n}\left(\mathbb{Z}\left[x_{1}, \ldots, x_{k}\right]\right.$ ) (where $n \geq 3$ ) is called the universal lattice by Y. Shalom in Sha1]. It was a long-standing problem to determine whether this group satisfies Kazhdan's property $(T)(\mathrm{Ka}])$, and finally Shalom and L. Vaserstein have answered this problem in the affirmative.

Theorem 1.1 (Shalom Sha4, Vaserstein Va]). Universal lattices $G=\mathrm{SL}_{n}\left(\mathbb{Z}\left[x_{1}\right.\right.$, $\left.\left.\ldots, x_{k}\right]\right)(n \geq 3)$ have property $(\mathrm{T})$.

Because property $(\mathrm{T})$ passes to group quotients, this result immediately implies that groups such as $\mathrm{SL}_{n \geq 3}\left(\mathbb{Z}[1 / p]\right.$ ) (here $p$ is a prime number), $\mathrm{SL}_{n \geq 3}(\mathbb{Z}[\sqrt{2}, \sqrt{3}]$ ), $\mathrm{SL}_{n \geq 3}\left(\mathbb{F}_{q}[x]\right)\left(\mathbb{F}_{q}\right.$ is the field of order $q$ and $q$ is a prime power $)$, and $\mathrm{SL}_{n \geq 3}\left(\mathbb{Z}\left[t, t^{-1}\right]\right)$ have property $(\mathrm{T})$. Note that in the four examples above, all but the last one are higher rank (hence arithmetic (or $S$-arithmetic)) lattices. Kazhdan's original result $\mathrm{Ka}$ states that higher rank lattices (recall the notation in this paper from the first paragraph) have property $(\mathrm{T})$. Hence in these cases property $(\mathrm{T})$ is classical. However the last example above cannot be realized as a higher rank (hence arithmetic)

Received by the editors October 22, 2010 and, in revised form, June 7, 2011.

2010 Mathematics Subject Classification. Primary 20F65, 20J06; Secondary 20H25, 22 D12.

Key words and phrases. Fixed point property, Kazhdan's property (T), Schatten class operators, noncommutative $L^{p}$-spaces, bounded cohomology.

The author is supported by JSPS Research Fellowships for Young Scientists No. 20-8313. 
lattice because it contains an infinite normal subgroup of infinite index and it violates the Margulis Normal Subgroup Theorem for higher rank lattices. Therefore property $(\mathrm{T})$ for this group had not been obtained before the Shalom-Vaserstein theorem, and property $(\mathrm{T})$ for universal lattices can be regarded as a nonarithmetization of extreme rigidity for higher rank lattices. Recall that the celebrated Delorme-Guichardet theorem (see [BHV]) states that property (T) is equivalent to property $(F H)$, which is defined as follows: a group $\Lambda$ is said to have property $(F H)$ if every (continuous) affine isometric action of $\Lambda$ on a Hilbert space has a global fixed point.

From this point, we always assume $p \in(1, \infty)$. In 2007, Bader, Furman, Gelander, and Monod considered fixed point properties in a much wider framework, and they defined the fixed point property, property $\left(F_{\mathcal{B}}\right)$, for a family $\mathcal{B}$ of Banach spaces (or a single Banach space $B$ ). They in particular paid heavy attention to the case of $\mathcal{B}=\mathcal{L}^{p}$, which denotes the family of all $L^{p}$-spaces, because property $\left(\mathrm{F}_{\mathcal{L}^{p}}\right)$ turns out to be much stronger than property $(\mathrm{T})\left(\Leftrightarrow\right.$ property $\left.(\mathrm{FH})=\left(\mathrm{F}_{\mathcal{L}^{2}}\right)\right)$, provided that $p \gg 2$. Indeed, $\mathrm{P}$. Pansu $\left[\mathrm{Pa}\right.$ ] showed that the group $\mathrm{Sp}_{n, 1}$, which has property $(\mathrm{T})$ if $n \geq 2$, fails to have property $\left(\mathrm{F}_{\mathcal{L}^{p}}\right)$ if $p>4 n+2$. This result is generalized by M. Bourdon and H. Pajot $[\mathrm{BP}]$ such that any hyperbolic group fails to have property $\left(\mathrm{F}_{\mathcal{L}^{p}}\right)$ if $p>1$ is strictly larger than the conformal dimension of the ideal boundary of the group. Moreover $\mathrm{G}$. $\mathrm{Yu}[\mathrm{Yu}]$ has shown that every hyperbolic group $H$, including a wide range of groups with property $(\mathrm{T})$, has $p>2$ such that it admits a metrically proper (that is, each orbit going to infinity as $h \in H$ goes to infinity) affine isometric action on $l^{p}$-space. In comparison, one of the main results of Bader-Furman-Gelander-Monod in [BFGM] is the following:

Theorem 1.2 ([BFGM $])$. Higher rank lattices, in the notation of this paper, have property $\left(\mathrm{F}_{\mathcal{L}^{p}}\right)$ for all $p \in(1, \infty)$.

In Mi], the author extended the Shalom-Vaserstein theorem and obtained the following "nonarithmetization" of Theorem 1.2

Theorem 1.3 (Mi]). Let $n \geq 4$. Then for any $p \in(1, \infty)$ the universal lattice $G=\mathrm{SL}_{n}\left(\mathbb{Z}\left[x_{1}, \ldots, x_{k}\right]\right)$ has property $\left(\mathrm{F}_{\mathcal{L}^{p}}\right)$.

Note that property $\left(\mathrm{F}_{\mathcal{L}^{p}}\right)$ is of high importance also from the viewpoint of group actions on the circle; see [Na, §4]. Also we mention, in Theorem 1.3, that the case of $n=3$ seems to remain open.

The main result of this paper is to extend this result to that for the family $\mathcal{C}_{p}$ of the spaces $C_{p}$ of $p$-Schatten class operators on any separable Hilbert space. Here for a separable Hilbert space $\mathfrak{H}$, a bounded linear operator $a \in \mathbb{B}(\mathfrak{H})$ is said to be of $p$-Schatten class $\left(a \in C_{p}\right)$ if $\operatorname{Tr}\left(|a|^{p}\right)<\infty$ holds, where $\operatorname{Tr}$ is the canonical (semifinite) trace: take an orthonomal basis $\left(\xi_{i}\right)_{i \in \mathbb{N}}$ for $\mathfrak{H}$, and for $t \in \mathbb{B}(\mathfrak{H})^{+}$, set

$$
\operatorname{Tr}(t):=\sum_{i \in \mathbb{N}}\left\langle t \xi_{i} \mid \xi_{i}\right\rangle \in[0, \infty]
$$

(which is independently determined by the choice of an orthonormal basis of $\mathfrak{H}$ ); $|a|:=\left(a^{*} a\right)^{1 / 2}$ is the absolute value of $a$. It can be regarded as a generalization of Theorem 1.3 to a noncommutative $L^{p}$-setting. The motivating fact in this study is that the fixed point property on $\mathcal{C}_{p}$ has the potential for some applications to group actions on higher-dimensional manifolds. 
Furthermore, in this paper, we consider a stronger property than property $\left(\mathrm{F}_{B}\right)$, called property $\left(F F_{B}\right)$. Recall that every affine isometric action $\alpha: G \curvearrowright B$ can be written as $\alpha(g) \cdot \xi=\rho(g) \xi+b(g)$, where $\rho$ is an isometric linear representation and $b: G \rightarrow B$ is a $\rho$-(1-) cocycle; namely, for any $g, h \in G, b(g h)=b(g)+\rho(g) b(h)$ holds. We consider a "quasification" of cocycles; namely, we allow uniformly bounded errors from being cocycles. Property $\left(\mathrm{FF}_{B}\right)$ is the boundedness property for any quasi-cocycle into every isometric representation in $B$. Property $\left(\mathrm{FF}_{B}\right) / \mathrm{T}$ is a weaker form of $\left(\mathrm{FF}_{B}\right)$ that asserts the boundedness of quasi-cocycles modulo the trivial linear part. Now we state our main result:

Theorem 1.4. Let $n \geq 4$. Then for any $p \in(1, \infty)$ the universal lattice $G=$ $\mathrm{SL}_{n}\left(\mathbb{Z}\left[x_{1}, \ldots, x_{k}\right]\right)$ has property $\left(\mathrm{F}_{\mathcal{C}_{p}}\right)$. Equivalently, every affine isometric action of $G$ on the space $C_{p}$ of $p$-Schatten class operators (on any separable Hilbert space) has a global fixed point. Furthermore, for any $p \in(1, \infty), G$ has property $\left(\mathrm{FF}_{\mathcal{C}_{p}}\right) / \mathrm{T}$ ("property $\left(\mathrm{FF}_{\mathcal{C}_{p}}\right)$ modulo the trivial part"). In particular, for any isometric linear representation $\rho$ on $C_{p}$ which satisfies $\rho \nsupseteq 1_{G}$, every quasi- $\rho$-cocycle is bounded.

Both property $\left(\mathrm{F}_{\mathcal{C}_{p}}\right)$ and property $\left(\mathrm{FF}_{\mathcal{C}_{p}}\right) / \mathrm{T}$ remain valid by taking group quotients and taking finite index subgroups of $G$ above.

For precise definitions of property $\left(\mathrm{FF}_{B}\right)$ and property $\left(\mathrm{FF}_{B}\right) / \mathrm{T}$, see Section 2 In the scope of the author, it seems unknown at the moment whether quasi-cocycles on universal lattices into the trivial representation must be bounded. Neither does the author know whether $\left(\mathrm{FF}_{B}\right) / \mathrm{T}$ is strictly weaker than $\left(\mathrm{FF}_{B}\right)$.

Therefore for any commutative and finitely generated ring $R$ (we always assume a ring $R$ is unital and associative), the following holds: the elementary group $\mathrm{E}_{n \geq 4}(R)$ and finite index subgroups therein have $\left(\mathrm{F}_{\mathcal{C}_{p}}\right)$, and property $\left(\mathrm{FF}_{\mathcal{C}_{p}}\right) / \mathrm{T}$. Here the elementary group over $R$ is the multiplicative group in the $n \times n$ matrix $\operatorname{ring} M_{n}(R)$ generated by elementary matrices. An elementary matrix in $M_{n}(R)$ is an $n \times n$ matrix whose entries are 1 on the diagonal and all but one entry off the diagonal are 0 . The Suslin stability theorem $([\mathrm{Su}])$ states that for $A=\mathbb{Z}\left[x_{1}, \ldots, x_{k}\right], \mathrm{E}_{n}(A)$ coincides with $\mathrm{SL}_{n}(A)$ provided $n \geq 3$, whereas $\mathrm{E}_{2}(A)$ is a proper subgroup of $\mathrm{SL}_{2}(A)([\mathrm{Co}])$.

Property $\left(\mathrm{F}_{B}\right)$ and Property $\left(\mathrm{FF}_{B}\right)$ have a natural interpretation in terms of (ordinary and bounded) group cohomology. Thus by Theorem 1.4, we have the following corollary. Here bounded cohomology is defined by requesting the additional condition that every cochain has bounded range, and the map $\Psi^{2}$ below is induced by the natural inclusion from bounded to ordinary cochain complexes ( $\Psi^{2}$ is called the comparison map in degree 2). For details of bounded cohomology with Banach coefficients, see [Mo, BM1, and BM2].

Corollary 1.5. Let $n \geq 4$ and let $R$ be a (unital, associative) commutative and finitely generated ring. Then for any $p \in(1, \infty)$, every finite index subgroup $\Gamma$ in $\mathrm{E}_{n}(R)$ satisfies the following:

- for any isometric linear representation $\rho$ on $C_{p}, H^{1}\left(\Gamma ; C_{p}, \rho\right)=0$;

- for any isometric linear representation $\rho$ on $C_{p}$ which satisfies $\rho \not \supset 1_{\Gamma}$, the natural map from second bounded cohomology to second cohomology:

$$
\Psi^{2}: H_{\mathrm{b}}^{2}\left(\Gamma ; C_{p}, \rho\right) \rightarrow H^{2}\left(\Gamma ; C_{p}, \rho\right)
$$

is injective. 
The latter item follows from the fact that the kernel of $\Psi^{2}$ above is naturally isomorphic to the following space:

$\{$ quasi- $\rho$-cocycles $\} /(\{\rho$-cocycles $\}+\{$ bounded maps $\})$.

Our proof of Theorem 1.4 for universal lattices (and group quotients) consists of two steps: in the first step, in Section 2 we show certain criteria for a family $\mathcal{B}$ of Banach spaces with respect to which universal lattices satisfy property $\left(\mathrm{F}_{\mathcal{B}}\right)$ and property $\left(\mathrm{FF}_{\mathcal{B}}\right) / \mathrm{T}$. To the best of the author's knowledge, no such criteria had been observed. Since our criteria seems to be of their own interest and importance, we state it here. For the definition of (relative) property $\left(\mathrm{T}_{\mathcal{B}}\right)$ and of ultraproducts of Banach spaces, we refer to Section 2 .

Theorem 1.6 (Criteria for fixed point properties for universal lattices). Set $A=$ $\mathbb{Z}\left[x_{1}, \ldots, x_{k}\right]$. Let $\mathcal{B}$ be a family of superreflexive Banach spaces. Suppose either of the following two conditions is fulfilled:

(i) The pair $\mathrm{E}_{2}(A) \ltimes A^{2} \unrhd A^{2}$ has relative property $\left(\mathrm{T}_{\mathcal{B}}\right)$, and $\mathcal{B}$ is stable under ultraproducts.

(ii) As properties for countable discrete groups, relative property (T) implies relative property $\left(\mathrm{T}_{\mathcal{B}}\right)$ : that means, for any pair of a countable discrete group $\Lambda$ and a normal (not necessarily proper) subgroup $\Lambda_{0}$ therein, whenever the pair $\Lambda \unrhd \Lambda_{0}$ has relative property $(\mathrm{T})$, it has relative property $\left(\mathrm{T}_{\mathcal{B}}\right)$.

Then for any $n \geq 4$, the universal lattice $\mathrm{SL}_{n}(A)$ possesses property $\left(\mathrm{F}_{\mathcal{B}}\right)$ and furthermore possesses property $\left(\mathrm{FF}_{\mathcal{B}}\right) / \mathrm{T}$.

Note that "condition ( $i i)$ implies the conclusion" deeply relies on Theorem 1.1. Also, if condition $(i i)$ is satisfied, then relative property $\left(\mathrm{T}_{\mathcal{B}}\right)$ for $\mathrm{E}_{2}(A) \ltimes A^{2} \unrhd A^{2}$ is automatic because relative property $(\mathrm{T})$ for that pair was shown in [Sha1] much earlier. Here by $\Lambda:=\mathrm{E}_{2}(A) \ltimes A^{2} \unrhd A^{2}=: \Lambda_{0}$ we mean

$$
\Lambda=\left\{(Z, \zeta)=\left(\begin{array}{c|c}
Z & \zeta \\
\hline 00 & 1
\end{array}\right): Z \in \mathrm{E}_{2}(A), \zeta \in A^{2}\right\} \unrhd\left\{\left(I_{2}, \zeta\right): \zeta \in A^{2}\right\}=\Lambda_{0} .
$$

For some examples of $\mathcal{B}$ which satisfy condition $(i)$ or condition $(i i)$, see Section 5 and the last part of Section 2 ,

In the second step, we verify that the family $\mathcal{C}_{p}$ indeed fulfills condition $(i i)$ :

Proposition 1.7. For any $p \in(1, \infty)$, relative property $(\mathrm{T})$ implies relative property $\left(\mathrm{T}_{\mathcal{C}_{p}}\right)$ among locally compact and second countable groups.

This part is inspired by a work of M. Puschnigg $[\mathrm{Pu}$, who extended a method of Bader-Furman-Gelander-Monod for the case of commutative $L^{p}$-spaces on $\sigma$-finite measures.

For the proof of Theorem 1.4 for finite index subgroups of universal lattices, we need the $p$-induction theory for (quasi)-1-cocycles of Bader-Furman-GelanderMonod. We also need to deal with the noncommutative $L^{p}$-space associated with the von Neumann algebra $L^{\infty}(\mathcal{D}) \bar{\otimes} \mathbb{B}(\mathfrak{H})$ (with the canonical trace), where $\mathcal{D}$ is a standard finite measure space. We examine them in Section 4 . The arguments above in addition imply the following:

Theorem 1.8. Higher rank lattices, in the notation of this paper, have property $\left(\mathrm{F}_{\mathcal{C}_{p}}\right)$ for all $p \in(1, \infty)$. More precisely, the following holds true: Let $G=$ $\prod_{i=1}^{m} \mathbf{G}_{i}\left(k_{i}\right)$, where $k_{i}$ are local fields and $\mathbf{G}_{i}\left(k_{i}\right)$ are $k_{i}$-points of Zariski connected 
simple $k_{i}$-algebraic groups $\mathbf{G}_{i}$. Assume each simple factor $\mathbf{G}_{i}\left(k_{i}\right)$ has $k_{i}$-rank at least 2 . Then $G$ and the lattices therein have property $\left(\mathrm{F}_{\mathcal{C}_{p}}\right)$ for any $p \in(1, \infty)$.

This result is a noncommutative analogue of Theorem 1.2 and can be seen to be a generalization of (a part of) a work of Puschnigg [Pu, Corollary 5.10]. For noncommutative $L^{p}$-spaces associated with semifinite von Neumann algebras other than $C_{p}$ (associated with $(\mathbb{B}(\mathfrak{H}), T r)$ ), see Remark 5.2, See also the remark below.

Organization of this paper: Section 2 is devoted to basic definitions and the proof of Theorem 1.6. In Section 3, we prove Proposition 1.7 and thus prove Theorem 1.4 for universal lattices. In Section 4 , we consider $p$-inductions and a generalization of Proposition 1.7. By these, we complete the proof of Theorem 1.4 and Theorem 1.8, Corollary 1.5] is immediate from Theorem 1.4 and a mere interpretation (see [BHV], [BFGM], [BM1], BM2, and [Mo]), so that we will not exhibit a proof of that. In Section [5] we make some concluding remarks on condition $(i)$ in Theorem 1.6.

Added Remark. Recently, in his work [O] independent of the author, B. Olivier has shown the considerably generalized versions of Proposition 1.7 and Theorem 1.8, more precisely, to the cases of noncommutative $L^{p}$-spaces associated with general (including type III cases) von Neumann algebras. By combining his result with item (ii) of Theorem 1.6, we obtain the fixed point property for universal lattices in degree at least 4 on general noncommutative $L^{p}$-spaces. The author is grateful to Baptiste Olivier and the referee of this paper for letting him know about the work above.

\section{Properties $\left(\mathrm{T}_{B}\right),\left(\mathrm{F}_{B}\right),\left(\mathrm{FF}_{B}\right),\left(\mathrm{FF}_{B}\right) / \mathrm{T}$, AND OUR CRITERIA}

Hereafter, we assume all Banach spaces $B$ in this paper are superreflexive. This condition is equivalent to $B$ having a compatible norm to a uniformly convex and uniformly smooth norm. A basic example is any $L^{p}$-space with $p \in(1, \infty)$. We refer to [BL, $\S \mathrm{A}]$ for a comprehensive study of this topic. In this section, $\Lambda$ is a locally compact second countable group and $\rho$ is a (continuous) isometric linear representation of $\Lambda$ in $B$.

Here we recall some basic results from BFGM, §2]: first, thanks to the superreflexivity of $B$, there exists a uniformly convex and uniformly smooth norm on $B$, compatible to the original norm, with respect to which $\rho$ is still isometric. Hence we can assume that $B$ is uniformly convex and uniformly smooth. Second, by the uniform smoothness, there is a natural complement $B_{\rho(\Lambda)}^{\prime}$ of $B^{\rho(\Lambda)}$ in $B$ which is a $\rho(\Lambda)$-invariant space. Here $B^{\rho(\Lambda)}$ is the subspace of $B$ of $\rho(\Lambda)$-invariant vectors. Also, $B_{\rho(\Lambda)}^{\prime}$ is isomorphic to $B / B^{\rho(\Lambda)}$ as $\rho(\Lambda)$ representation spaces. Precisely, $B_{\rho(\Lambda)}^{\prime}$ is the annihilator of the subspace $\left(B^{*}\right)^{\rho^{\dagger}(\Lambda)} \subseteq B^{*}$ in $B$, where $\rho^{\dagger}$ denotes the contragradient representation: for the duality $\langle\cdot, \cdot\rangle: B \times B^{*} \rightarrow \mathbb{C}$ and for any $g \in \Lambda$, any $x \in B$, and any $\phi \in B^{*},\left\langle x, \rho^{\dagger}(g) \phi\right\rangle:=\left\langle\rho\left(g^{-1}\right) x, \phi\right\rangle$. Finally, by uniform convexity of $B$, for any (continuous) affine isometric action, the existence of a $\Lambda$-fixed point is equivalent to boundedness of some (equivalently, any) $\Lambda$-orbit. By considering an orbit of the origin $0 \in B$, this means that for any $\rho$ on $B$, a $\rho(-1)$-cocycle $b$ is a coboundary if and only if it has bounded range.

Now we recall the definitions of property $\left(\mathrm{T}_{B}\right)$ and property $\left(\mathrm{F}_{B}\right)$ in [BFGM], and property $\left(\mathrm{FF}_{B}\right)$ and property $\left(\mathrm{FF}_{B}\right) / \mathrm{T}$ in [Mi]. Note that if $\Lambda_{0} \unlhd \Lambda$ is a normal subgroup, then $B=B^{\rho\left(\Lambda_{0}\right)} \oplus B_{\rho\left(\Lambda_{0}\right)}^{\prime}$ can be seen as a decomposition of $B$ as $\Lambda$-representation spaces. 
Definition 2.1. Let $\Lambda, B, \rho$ be as in the setting of this section.

- The representation $\rho$ of $\Lambda$ on $B$ is said to have almost invariant vectors, written as $\rho \succ 1_{\Lambda}$, if the following holds: for any compact subset $K \subset \Lambda$, there exists a sequence of unit vectors $\left(\xi_{n}\right)_{n}$ in $B$ such that $\max _{s \in K} \| \xi_{n}-$ $\rho(s) \xi_{n} \| \rightarrow 0$ as $n \rightarrow \infty$.

- A continuous map $b: \Lambda \rightarrow B$ is called a $\rho$-cocycle if for any $g, h \in \Lambda$, $b(g h)=b(g)+\rho(g) b(h)$ holds. The map $b$ is called a quasi- $\rho$-cocycle if $\sup _{g, h}\|b(g h)-b(g)-\rho(g) b(h)\|<\infty$ holds.

Definition 2.2 ([BFGM], $\mathrm{Mi}]$ ). Let $\Lambda, B, \rho$ be as in the setting of this section, and fix $B$.

(1) A group pair $\Lambda \unrhd \Lambda_{0}$ is said to have relative property $\left(T_{B}\right)$ if the following holds true: for any $\rho$ of $\Gamma$ on $B$, the isometric linear representation $\rho^{\prime}$, constructed by restricting $\rho$ on $B_{\rho\left(\Lambda_{0}\right)}^{\prime}, \rho^{\prime}: \Lambda \rightarrow O\left(B_{\rho\left(\Lambda_{0}\right)}^{\prime}\right)$ does not have almost invariant vectors. The group $\Lambda$ is said to have property $\left(T_{B}\right)$ if $\Lambda \unrhd \Lambda$ has relative property $\left(\mathrm{T}_{B}\right)$.

(2) A group pair $\Lambda \geqslant \Lambda_{0}$ is said to have relative property $\left(F_{B}\right)$ if for any $\rho$ of $\Lambda$ on $B$, every $\rho$-cocycle $b$ is a coboundary on the subgroup $\Lambda_{0}$, namely, if there exists $\xi \in B$ such that for any $h \in \Lambda_{0}, b(h)=\xi-\rho(h) \xi$ holds. Equivalently, $b$ is bounded on $\Lambda_{0}$. The group $\Lambda$ is said to have property $\left(F_{B}\right)$ if $\Lambda \geqslant \Lambda$ has relative property $\left(\mathrm{F}_{B}\right)$.

(3) A group pair $\Lambda \geqslant \Lambda_{0}$ is said to have relative property $\left(F F_{B}\right)$ if for any $\rho$ of $\Lambda$ on $B$, every (continuous) quasi- $\rho$-cocycle $b$ is bounded on $\Lambda_{0}$. The group $\Lambda$ is said to have property $\left(F F_{B}\right)$ if $\Lambda \geqslant \Lambda$ has relative property $\left(\mathrm{FF}_{B}\right)$.

(4) The group $\Lambda$ is said to have property $\left(F F_{B}\right) / T$ (which means property $\left(F F_{B}\right)$ modulo the trivial part) if for any $\rho$ of $\Lambda$ on $B$ and any (continuous) quasi$\rho$-cocycle $b, b^{\prime}(\Lambda)$ is bounded. Here $b^{\prime}: \Lambda \rightarrow B_{\rho(\Lambda)}^{\prime}$ means the projection of $b$ to a quasi- $\rho^{\prime}$-cocycle which ranges in $B_{\rho(\Lambda)}^{\prime}$, associated with the decomposition $B=B^{\rho(\Lambda)} \oplus B_{\rho(\Lambda)}^{\prime}$.

If $B=\mathcal{B}$ is a family of Banach spaces, these seven properties are defined as having corresponding properties for all Banach spaces $E \in \mathcal{B}$.

We make two remarks on the definition above: first, if $B$ is a general (possibly not superreflexive) Banach space, $B_{\rho(\Lambda)}^{\prime}$ is replaced with $B / B^{\rho(\Lambda)}$ in the definitions of (relative) $\left(\mathrm{T}_{B}\right)$ and $\left(\mathrm{FF}_{B}\right) / \mathrm{T}$. Secondly, if $\Lambda$ has a compact abelianization, then property $\left(\mathrm{FF}_{B}\right) / \mathrm{T}$ implies property $\left(\mathrm{F}_{B}\right)$. In particular, the following holds true:

Lemma 2.3. For any $p \in(1, \infty)$, property $\left(\mathrm{FF}_{\mathcal{L}^{p}}\right) / \mathrm{T}$ implies property $\left(\mathrm{F}_{\mathcal{L}^{p}}\right)$.

Proof. Let $\Lambda$ have $\left(\mathrm{FF}_{\mathcal{L}^{p}}\right) / \mathrm{T}$. Suppose $H:=\Lambda / \overline{[\Lambda, \Lambda]}$ is noncompact. Then consider the $\left(L^{p}\right.$-)left regular representation $\lambda_{H}$ of $H$ in $L^{p}(H)$ and a representation $\rho$ of $\Lambda$ which is the pull-back of $\lambda_{H}$ by $\Lambda \rightarrow H$. Since $H$ is abelian and noncompact, $\lambda_{H} \succ 1_{H}$ but $\lambda_{H} \not \supset 1_{H}$. Therefore there exists a $\lambda_{H}$-cocycle which is not a coboundary (for details, see [BFGM, §3.a]). Thus we have a $\rho$-cocycle which is not a coboundary. However this contradicts $\rho \not \supset 1_{\Lambda}$ and $\left(\mathrm{FF}_{\mathcal{L}_{p}}\right) / \mathrm{T}$ for $\Lambda$.

By the Delorme-Guichardet theorem, if $\mathcal{B}$ is the family $\mathcal{H}$ of all Hilbert spaces $\left(=\mathcal{L}^{2}\right)$, then property $\left(\mathrm{F}_{\mathcal{H}}\right)=(\mathrm{FH})$ is equivalent to property $\left(\mathrm{T}_{\mathcal{H}}\right)=(\mathrm{T})$. We note that in the spirit of this, property $\left(\mathrm{FF}_{\mathcal{H}}\right)$ was previously defined and called 
property $(T T)$ by N. Monod $\left[\mathrm{Mo}\right.$. In the same spirit, we also call $\left(\mathrm{FF}_{\mathcal{H}}\right) / \mathrm{T}$ property $(T T) / T$. By Lemma 2.3, (TT)/T implies (T).

We now explain one more concept which appears in Theorem 1.6, namely, an ultraproduct of Banach spaces. For a precise definition and comprehensive treatment, see $[\mathrm{He}$. Here we briefly recall the definition: Take a nonprincipal ultrafilter $\omega$ on $\mathbb{N}$ and fix it. For a sequence $\left(\left(B_{n},\|\cdot\|_{n}\right)\right)_{n}$ of pairs of a Banach space and the norm, we define the ultraproduct $\lim _{\omega} B_{n}=\left(B_{\omega},\|\cdot\|_{\omega}\right)$ as follows: we define $B_{\omega}$ as $\left(\bigoplus_{n \in \mathbb{N}}\left(B_{n},\|\cdot\|_{n}\right)\right)_{\infty} / \mathcal{N}$. Here $\left(\bigoplus_{n \in \mathbb{N}}\left(B_{n},\|\cdot\|_{n}\right)\right)_{\infty}$ means the set of sequences with bounded norms. We define a seminorm $\|\cdot\|$ there by $\left\|\left(\xi_{n}\right)_{n}\right\|:=\lim _{\omega}\left\|\xi_{n}\right\|_{n}$ and set $\mathcal{N}$ as the null subspace with respect to $\|\cdot\|$. Finally, define a norm $\|\cdot\|_{\omega}$ on $B_{\omega}$ as the induced norm by $\|\cdot\|$ above. We say that a family $\mathcal{B}$ is stable under ultraproducts if whenever $B_{n} \in \mathcal{B}$ for all $n \in \mathbb{N}$, $\lim _{\omega} B_{n} \in \mathcal{B}$ holds.

Before proving Theorem 1.6, we make a remark that "condition $(i)$ implies the conclusion" has the spirit of the original strategy of Shalom Sha4 to prove Theorem 1.1 and of an observation by M. Gromov. Precisely, for the proof of this part, one essential part is to use reduced-1-cohomology. The original argument in Sha2 uses conditionally negative definite functions, but Gromov $\mathrm{Gr}$ observed that it can be done in terms of scaling limits on a metric space, which are special cases of ultraproducts for Banach spaces. For details, see [Mi, §5] for instance.

Proof of Theorem 1.6. The proof follows from a combination of previous results in Mi]. Keeping the same notation as in Theorem 1.6, we list necessary results:

Theorem 2.4 ([Mi, Theorem 1.3, Theorem 6.4]). Let $B$ be a superreflexive space or a family of them. Suppose $\mathrm{E}_{2}(A) \ltimes A^{2} \unrhd A^{2}$ has relative property $\left(\mathrm{T}_{B}\right)$. Then for any $m \geq 3, \mathrm{SL}_{m}(A) \ltimes A^{m} \geqslant A^{m}$ has relative property $\left(\mathrm{F}_{B}\right)$. In fact, this pair has relative property $\left(\mathrm{FF}_{B}\right)$.

Theorem 2.5 ([Mi, Theorem 5.5], Shalom's machinery). Let $\mathcal{B}$ be a family of superreflexive Banach spaces and $n \geq 3$. Suppose $\mathrm{SL}_{n}(A) \geqslant A^{n-1}$ has relative property $\left(\mathrm{F}_{\mathcal{B}}\right)$, where $A^{n-1}$ sits on a unipotent part, from $(1, n)$-th to $(n-1, n)$-th entries. If $\mathcal{B}$ is moreover stable under ultraproducts, then $\mathrm{SL}_{n}(A)$ possesses property $\left(\mathrm{F}_{\mathcal{B}}\right)$.

Proposition 2.6 ([Mi, Proposition 6.6]). Let B be a superreflexive Banach space or a family of them, and let $n \geq 3$. Suppose $\mathrm{SL}_{n}(A) \geqslant A^{n-1}$ has relative property $\left(\mathrm{FF}_{B}\right)$, where $A^{n-1}$ sits in the same way as Theorem 2.5. If $\mathrm{SL}_{n}(A)$ moreover satisfies property $\left(\mathrm{T}_{B}\right)$, then $\mathrm{SL}_{n}(A)$ possesses property $\left(\mathrm{FF}_{B}\right) / \mathrm{T}$.

Indeed, for Theorem 2.5 and Proposition 2.6. we need a quadruple $\left(\Lambda, \Lambda^{\prime}, H_{1}, H_{2}\right)$ of a countable discrete group $\Lambda$ with finite abelianization and subgroups $\Lambda^{\prime}, H_{1}, H_{2}$ therein satisfying the following three conditions:

(1) The group $\Lambda$ is generated by $H_{1}$ and $H_{2}$ together.

(2) The subgroup $\Lambda^{\prime}$ normalizes $H_{1}$ and $H_{2}$.

(3) The group $\Lambda$ is boundedly generated by $\Lambda^{\prime}, H_{1}$, and $H_{2}$.

Here we say that a subset $\mathcal{S} \subset \Lambda$ containing the unit $e \in \Lambda$ boundedly generates a group $\Lambda$ if there exists $N \in \mathbb{Z}_{>0}$ such that $\mathcal{S}^{N}=\Lambda$ holds (this equality means that any $g \in \Lambda$ can be expressed as a product of $N$ elements in $\mathcal{S}$ ). We warn that in some 
other literature, the terminology bounded generation is used only for the following confined case: $\mathcal{S}$ is a finite union of cyclic subgroups of $\Lambda$. These properties relate to some forms of Shalom properties, which are used in the proofs of Theorem 2.5 and Proposition 2.6. For more details, compare [Mi, Definition 5.4, Definition 6.5].

We get the last two results stated above by letting $\left(\Lambda, \Lambda^{\prime}, H_{1}, H_{2}\right)$ in the original statements be $\left(\mathrm{SL}_{n}(A), \mathrm{SL}_{n-1}(A), A^{n-1}, A^{n-1}\right)$. Here $\Lambda^{\prime}$ sits in the upper left corner of $\mathrm{SL}_{n}(A), H_{1} \cong A^{n-1}$ sits in $\mathrm{SL}_{n}(A)$ as a unipotent part, from $(1, n)$-th to $(n-1, n)$-th entries, and $H_{2} \cong A^{n-1}$ sits in $\mathrm{SL}_{n}(A)$ as a unipotent part, from $(n, 1)$-th to $(n, n-1)$-th entries. Condition (3) for this case follows from a deep theorem of Vaserstein $\mathrm{Va}$.

First, we deal with the case in which condition $(i)$ is fulfilled. Then by combining Theorem 2.4 and Theorem 2.5, we obtain that $\mathrm{SL}_{n \geq 4}(A)$ has property $\left(\mathrm{F}_{\mathcal{B}}\right)$ (note that $\mathrm{SL}_{m}(A) \ltimes A^{m}$ naturally injects into $\mathrm{SL}_{m+1}(A)$ ). Since property $\left(\mathrm{F}_{\mathcal{B}}\right)$ implies property $\left(\mathrm{T}_{\mathcal{B}}\right)$ (it is a general fact; see [BFGM, Theorem 1.3]), $\mathrm{SL}_{n \geq 4}(A)$ has property $\left(\mathrm{T}_{\mathcal{B}}\right)$. Then by Proposition 2.6 , we obtain property $\left(\mathrm{FF}_{\mathcal{B}}\right) / \mathrm{T}$ as well.

Second, we consider the case in which condition $(i i)$ is satisfied. In general, we may not apply Theorem 2.5. However, in this case, we can first apply Theorem 2.4 and next appeal directly to Proposition 2.6. The point here is that since relative (T) implies relative $\left(\mathrm{T}_{\mathcal{B}}\right)$, the pairs $\mathrm{E}_{2}(A) \ltimes A^{2} \unrhd A^{2}$ and $\mathrm{SL}_{n}(A) \unrhd \operatorname{SL}_{n}(A)(n \geq$ 3) have relative $\left(\mathrm{T}_{\mathcal{B}}\right)$ (these respectively follow from [Sha1] and Theorem 1.1). Thus for $\mathrm{SL}_{n \geq 4}(A)$, we obtain property $\left(\mathrm{FF}_{\mathcal{B}}\right) / \mathrm{T}$. Since $\mathrm{SL}_{n \geq 4}(A)$ has the trivial abelianization, we get $\left(\mathrm{F}_{\mathcal{B}}\right)$ as well.

We mention that Bader-Furman-Galander-Monod [BFGM, §4.a]) have shown that relative $(\mathrm{T})$ implies relative $\left(\mathrm{T}_{L^{p}(\mu)}\right)$ for any $\sigma$-finite measure $\mu$. Moreover, Heinrich $\left[\mathrm{He}\right.$ has proven that the family $\mathcal{L}^{p}$ satisfies condition $(i)$. Thus although we saw that property $\left(\mathrm{F}_{\mathcal{L}^{p}}\right)$ is stronger than property $(\mathrm{T})$ for $p$ large enough (recall this from the introduction), for universal lattices with $n \geq 4$ we even obtain property $\left(\mathrm{FF}_{\mathcal{L}^{p}}\right) / \mathrm{T}([\mathrm{Mi}$, Theorem 1.5]). Also in [Mi, Remark 6.7], we have obtained (TT)/T $\left(=\left(\mathrm{FF}_{\mathcal{L}^{2}}\right) / \mathrm{T}\right)$ for $\mathrm{SL}_{n \geq 3}(A)$. Note that $(\mathrm{TT}) / \mathrm{T}$ is strictly stronger than $(\mathrm{T})$ because any non-elementary hyperbolic group, including one with $(\mathrm{T})$, is known to admit an unbounded quasi-cocycle into the left regular representation; see [MMS.

In Section 3, we shall see that the family $\mathcal{C}_{p}$ satisfies condition $(i i)$. For a family $\mathcal{B}$ having condition $(i)$ but not satisfying condition $(i i)$, see Section 5 .

\section{Relative Property (T) implies Relative Property $\left(\mathrm{T}_{\mathcal{C}_{p}}\right)$}

We refer to $[\mathrm{PX}]$ for comprehensive treatments on noncommutative $L^{p}$-spaces including $C_{p}$. First of all, the Clarkson-type inequality (for instance, see [PX, §5]) implies that $C_{p}$ for any $p(\in(1, \infty))$ is uniformly convex and uniformly smooth.

Before proceeding to the proof of Proposition 1.7, we briefly recall the strategy in [BFGM, $\S 2 . e, \S 4$.a] of proving the following commutative version of this theorem: property $(\mathrm{T})$ implies property $\left(\mathrm{T}_{L^{p}(\mu)}\right)$ for a $\sigma$-finite measure space $\mu$. The keys to their proof are the following two tools:

Tool 1. (The Mazur map: interpolation between $L^{p}$-spaces; see $[\mathrm{BL}$ ) For $p, r \in$ $(1, \infty)$ and a $\sigma$-finite measure, the map $M_{p, r}: L^{p}(\mu) \rightarrow L^{r}(\mu) ; M_{p, r}(f)=$ $\operatorname{sign}(f) \cdot|f|^{p / r}$ is a (nonlinear) map, and this induces a uniformly continuous homeomorphism between the unit spheres $M_{p, r}: S\left(L^{p}(\mu)\right) \rightarrow S\left(L^{r}(\mu)\right)$. 
Tool 2. (The Banach-Lamperti theorem: classification of linear isometries on an $L^{p}$-space; see [FJ]). For any $1<p<\infty$ with $p \neq 2$, any linear isometry $V$ of $L^{p}(X, \mu)$ has the form

$$
V f(x)=f(F(x)) h(x)\left(\frac{d F_{*} \mu}{d \mu}(x)\right)^{\frac{1}{p}},
$$

where $F$ is a measurable, measure class preserving map of a Borel space $(X, \mu)$, and $h$ is a measurable function with $|h(x)|=1$ almost everywhere.

Their proof goes as follows: suppose that a group $\Lambda$ does not have property $\left(\mathrm{T}_{L^{p}(\mu)}\right)$. Then there exists a (continuous) isometric linear representation $\rho$ on $B=L^{p}(\mu)$ such that $\rho^{\prime} \succ 1_{\Lambda}$ (namely, $\rho^{\prime}$ has almost invariant vectors). Here $\rho^{\prime}$ is the restriction of $\rho$ on the subspace $B^{\prime}:=B_{\rho(\Lambda)}^{\prime}$; recall the definitions above from Section 2. Through Tool 1, define $\pi$ by $\pi(g)=M_{p, 2} \circ \rho(g) \circ M_{2, p}(g \in \Lambda)$. Then thanks to Tool 2, one can show that this $\pi$ maps each $g \in \Lambda$ to a linear (unitary) operator on the Hilbert space $\mathfrak{H}:=L^{2}(\mu)$. Thus one obtains the unitary representation $\pi: \Lambda \rightarrow \mathcal{U}(\mathfrak{H})$. Finally, by uniform continuity of the Mazur maps, it is not difficult to see that $\rho^{\prime} \succ 1_{\Lambda}$ implies $\pi^{\prime} \succ 1_{\Lambda}$ (where $\pi^{\prime}$ is the restriction of $\pi$ on the orthogonal complement of $\left.\mathfrak{H}^{\pi(\Lambda)}\right)$. This means that $\Lambda$ does not have property $(\mathrm{T})$. Hence property $(\mathrm{T})$ implies property $\left(\mathrm{T}_{L^{p}(\mu)}\right)$. Also in this argument one can reach the same conclusion for relative properties.

Proof of Proposition 1.7. Puschnigg $[\mathrm{Pu}$. showed a noncommutative analogue of Tool 1 for the case of a $p$-Schatten class $C_{p}$ :

Theorem 3.1 (Puschnigg $[\mathrm{Pu}$, Corollary 5.6]). Let $1<p, r<\infty$. Then the noncommutative Mazur map $M_{p, r}: S\left(C_{p}\right) \rightarrow S\left(C_{r}\right) ; a \mapsto u \cdot|a|^{p / r}$ is a uniform continuous homeomorphism between unit spheres. Here $a=u \cdot|a|$ is a polar decomposition.

In $\left[\mathrm{Pu}\right.$, Puschnigg considered isometric linear representations on $C_{p}$ which come from unitary representations, and hence he did not need an analogue of Tool 2. For our case, it is needed and is obtained by J. Arazy $\underline{\mathrm{Ar}}$. Since our Hilbert space $\mathfrak{H}$ is separable, by choosing a countable orthonormal basis, we can identify $\mathfrak{H}$ with a square integrable sequence space $\ell^{2}$. Through this identification, we can consider the transpose map $a \mapsto^{T} a$ on $\mathbb{B}(\mathfrak{H}) \cong \mathbb{B}\left(\ell^{2}\right)$. Although the transpose map depends on the choices of bases, it is easy to obtain the following facts:

Lemma 3.2. Stick to the setting above.

(i) The transpose map is a linear isometry on each $C_{p}$.

(ii) The transpose map is compatible with the adjoint operation. Namely, for any $a \in \mathbb{B}\left(\ell^{2}\right),\left({ }^{T} a\right)^{*}={ }^{T}\left(a^{*}\right)$.

(iii) For any $a, b \in \mathbb{B}\left(\ell^{2}\right),{ }^{T}(a b)={ }^{T} b^{T} a$.

(iv) If $u$ is a unitary, then so is ${ }^{T} u$.

(v) If $t$ is positive and $\alpha>0$, then ${ }^{T} t$ is positive and ${ }^{T}\left(t^{\alpha}\right)=\left({ }^{T} t\right)^{\alpha}$.

Now we state the theorem of Arazy:

Theorem 3.3 (Arazy $\underline{\mathrm{Ar}}$ ). Let $1<p<\infty$ with $p \neq 2$ and $C_{p}$ be the space of $p$-Schatten class operators on $\ell^{2}$. Then every linear isometry $V$ on $C_{p}$ is either of the following two forms: 
(1) there exist unitaries $w, v \in \mathcal{U}\left(\ell^{2}\right)$ such that $V: a \mapsto w a v$;

(2) there exist unitaries $w, v \in \mathcal{U}\left(\ell^{2}\right)$ such that $V: a \mapsto w^{T}$ av.

Thanks to the two theorems above, by following the footsteps of Bader-FurmanGelander-Monod we accomplish the conclusion. Indeed, for linearity of the composition, let us take a linear isometry $V$ on $C_{p}$. By Lemma 3.2, for $\widetilde{V}=M_{p, 2} \circ$ $V \circ M_{2, p}: S\left(C_{2}\right) \rightarrow S\left(C_{2}\right)$ we have the following (recall that one can take a partial isometry in a polar decomposition as a unitary):

(i) In the case of (1), for any $x \in C_{2}$ with a polar decomposition $x=u|x|$, a polar decomposition of $w u|x|^{2 / p} v$ is $(w u v)\left(v^{*}|x|^{2 / p} v\right)$. Therefore, we have

$$
\begin{aligned}
\widetilde{V} \cdot x & =w u v\left(v^{*}|x|^{2 / p} v\right)^{p / 2}=w u v v^{*}|x| v \\
& =w u|x| v=w x v .
\end{aligned}
$$

(ii) In the case of (2), for any $x \in C_{2}$ with a polar decomposition $x=u|x|$, a polar decomposition of $w^{T}\left(u|x|^{2 / p}\right) v=w\left({ }^{T}|x|\right)^{2 / p}\left({ }^{T} u\right) v$ is

$$
\left(w^{T} u v\right)\left\{v^{*}\left({ }^{T} u\right)^{*}\left({ }^{T}|x|\right)^{2 / p}\left({ }^{T} u\right) v\right\} .
$$

Therefore, we have

$$
\begin{aligned}
\widetilde{V} \cdot x & =w^{T} u v\left\{v^{*}\left({ }^{T} u\right)^{*}\left({ }^{T}|t|\right)^{2 / p}\left({ }^{T} u\right) v\right\}^{p / 2} \\
& =w^{T} u v v^{*}\left({ }^{T} u\right)^{*}\left({ }^{T}|x|\right)^{T} u v \\
& =w^{T}|x|^{T} u v=w^{T}(u|x|) v=w\left({ }^{T} x\right) v .
\end{aligned}
$$

Hence in both cases $\widetilde{V}$ is linear. Now recall that $C_{2}$, the space of Hilbert-Schmidt operators, is indeed a Hilbert space equipped with the inner product: $\langle x \mid y\rangle:=$ $\operatorname{Tr}\left(y^{*} x\right)$ (this holds for noncommutative $L^{2}$ spaces in a general setting).

Proposition 1.7 together with Theorem 1.6 immediately implies Theorem 1.4 for universal lattices and group quotients of them. In Section 4 we deal with the case of finite index subgroups by utilizing $p$-inductions.

\section{Finite index SUbGroups, LAtTiCEs, AND $L^{p}$-INDUCTIONS}

The $p$-induction of (quasi-)cocycles is given by Shalom [Sha3, $\S 3$. III] for $p=2$, and later by Bader-Furman-Gelander-Monod [BFGM, §8] for general $p$.

Let $G$ be a (locally compact second countable) group, $\Gamma \leqslant G$ be a lattice, and $\mathcal{D}$ be a Borel fundamental domain for $\Gamma$ (namely, $\mathcal{D}$ is a Borel subset of $G$ such that $\left.G=\bigsqcup_{\gamma \in \Gamma} \mathcal{D} \gamma\right)$. For the existence of such a domain, see [BHV, $\left.\S \mathrm{B}\right]$. We let $\mu$ be a Haar measure of $G$ with $\mu(\mathcal{D})=1$, and we identify $\mathcal{D}$ with $G / \Gamma$ and regard $\mathcal{D}$ as a (left) $G$-space. We define a Borel cocycle $\beta: G \times \mathcal{D} \rightarrow \Gamma$ by the following rule:

$$
\beta(g, d)=\gamma \text { if and only if } g^{-1} d \gamma \in \mathcal{D} .
$$

Now for a given isometric $\Gamma$-representation $\sigma$ on a Banach space $B$ and a (quasi$\sigma$-cocycle $b: \Gamma \rightarrow B$, under some integrability condition as below, we can define the $p$-induction $\tilde{b}$ of $b$ as follows:

- the $p$-induced Banach space is $L^{p}(\mathcal{D}, B)$, the space of $p$-Bochner integrable functions; 
- the $p$-induced representation $\rho=\operatorname{Ind}_{\Gamma}^{G} \sigma$ in $L^{p}(\mathcal{D}, B)$ is defined as follows: for $g \in G, \xi \in L^{p}(\mathcal{D}, B)$ and $d \in \mathcal{D}$,

$$
\rho(g) \xi(d):=\sigma(\beta(g, d)) \xi\left(g^{-1} \cdot d\right)
$$

(recall that we have endowed $\mathcal{D}$ with a $G$-space structure);

- we define the $\tilde{b}: G \rightarrow L^{p}(\mathcal{D}, B)$ as

$$
\tilde{b}(g)(d):=b(\beta(g, d)) \quad(g \in G, d \in \mathcal{D}),
$$

provided that $\tilde{b}(g)$ is p-integrable for all $g \in G$.

Then under the condition above, this $\tilde{b}$ becomes a (quasi-) $\operatorname{Ind}_{\Gamma}^{G} \sigma$-cocycle.

Remark 4.1. The $p$-induction procedure of (quasi-)cocycles requires the integrable condition above, and in general it is a subtle problem to determine whether this holds. However, it is known that this condition is satisfied for all $p$ in the following two cases:

(1) if $\Gamma$ is cocompact in $G$, in particular, if $\Gamma$ is a finite index subgroup of (a countable) $G$, then for any $\mathcal{D}$ and any $b$ the integrability condition is fulfilled;

(2) if $G$ is a semisimple algebraic group with each simple factor having a local rank at most 2 , then there exists $\mathcal{D}$ such that for any $b$ the integrability condition is fulfilled.

For case (1), it is almost trivial to check the integrability. However for case (2), a proof of this fact is considerably involved: one needs a deep result on length functions on higher rank lattices [LMR; also see [Sha2, §2] on how to deduce the integrability from that result.

Now we state the strategy to prove Theorem 1.4 for finite index subgroups of universal lattices and also Theorem 1.8. At the beginning, we consider a general setting. Let $G$ be a (locally compact and second countable) group and $\Gamma$ be a lattice in $G$. Let $\sigma$ be an isometric $\Gamma$-representation in $C_{p}$. Take a Borel fundamental domain $(\mathcal{D}, \mu)$ of $\Gamma$ and consider the $p$-induction (with the same $p$ as $C_{p}$ ) $\rho=\operatorname{Ind}_{\Gamma}^{G} \sigma$ of $\sigma$ on $E=L^{p}\left(\mathcal{D}, C_{p}\right)$. Note that $E$ is identical to the following space:

$$
\left\{x: \mathcal{D} \rightarrow C_{p}:\|x\|_{p}^{p}:=\int_{d \in \mathcal{D}} \operatorname{Tr}\left(|x(d)|^{p}\right) d \mu<\infty\right\} .
$$

We need to have analogues of Tool 1 and Tool 2 in Section 3 . Note that $E=$ $L^{p}\left(\mathcal{D}, C_{p}\right)$ can be seen as the noncommutative $L^{p}$-space associated with the von Neumann algebra $L^{\infty}(\mathcal{D}, \mathbb{B}(\mathfrak{H})) \cong L^{\infty}(\mathcal{D}) \bar{\otimes} \mathbb{B}(\mathfrak{H})$ (acting on $\left.L^{2}(\mathcal{D}) \otimes \mathfrak{H}\right)$ with the canonical (normal faithful) semifinite trace $\tau=\tau_{L^{\infty}} \otimes \operatorname{Tr}$ : for $x \in L^{\infty}\left(\mathcal{D}, \mathbb{B}(\mathfrak{H})^{+}\right)$,

$$
\tau(x):=\int_{d \in \mathcal{D}} \operatorname{Tr}(x(d)) d \mu \in[0, \infty] .
$$

For the definition of a noncommutative $L^{p}$-space $L_{p}(\mathcal{A}, \tau)$ associated with a semifinite von Neumann algebra $(\mathcal{A}, \tau)$, see [PX]. We use this symbol to distinguish it from $L^{p}\left(\mathcal{D}, C_{p}\right)$ above. For Tool 1, we utilize the following result of Puschnigg:

Theorem 4.2 (Puschnigg $[\mathrm{Pu}$, Corollary 5.7]). For $1<p, r<\infty$, the analogue of the Mazur map (Theorem 3.1)

$$
\tilde{M}_{p, r}: L^{p}\left(\mathcal{D}, C_{p}\right) \rightarrow L^{r}\left(\mathcal{D}, C_{r}\right) ; x \mapsto u \cdot|x|^{p / r}
$$

is a uniform continuous homeomorphism on unit spheres. Here $x=u|x|$ is a polar decomposition. 
Note that the uniform convexity and the uniform smoothness of $E$ follow from a general theory; see $\mathrm{FP}$.

For Tool 2, F. J. Yeadon has shown the following theorem on isometries in the noncommutative $L^{p}$-space associated with a semifinite von Neumann algebra:

Theorem 4.3 (Yeadon [Ye, Theorem 2]). Let $\mathcal{A}$ be a von Neumann algebra with a faithful semifinite normal trace $\tau$ and let $L_{p}(\mathcal{A}, \tau)$ be the associated noncommutative $L^{p}$-space with $p \neq 2$ in $(1, \infty)$. Suppose $V$ is a linear isometry. Then there exist, uniquely, a partial isometry $w \in \mathcal{A}$, a normal Jordan $*$-monomorphism, and an (unbounded) positive self-adjoint operator $N$ affiliated with $\mathcal{A} \cap J(\mathcal{A})^{\prime}$ such that

- $w^{*} w=J(1)=$ the support projection of $N$;

- for any $x \in \mathcal{A}^{+}, \tau(x)=\tau\left(N^{p} J(x)\right)$;

- for any $x \in \mathcal{A} \cap L_{p}(\mathcal{A}, \tau), V \cdot x=w N J(x)$.

Here $\mathcal{A}^{\prime}$ denotes the commutant of $\mathcal{A}$, and a closed and densely defined operator $L$ is said to be affiliated with a von Neumann algebra $\mathcal{A}_{0}$ (both acting on the same Hilbert space) if every unitary $u$ in $\mathcal{A}_{0}^{\prime}$ carries the domain of $L$ onto itself and satisfies $u L u^{*}=L$ there. A Jordan $*$-monomorphism of a von Neumann algebra $\mathcal{A}$ is an injective linear map $J: \mathcal{A} \rightarrow \mathcal{A}$ which satisfies for any $x \in \mathcal{A}, J\left(x^{2}\right)=J(x)^{2}$.

With the aid of this theorem, we claim the following:

Proposition 4.4. For a standard finite measure space $\mathcal{D}$ and $p \neq 2$ in $(1, \infty)$, let $V$ be a linear isometry on $L^{p}\left(\mathcal{D}, C_{p}\right)$. Then the composition

$$
\tilde{V}=\tilde{M}_{p, 2} \circ V \circ \tilde{M}_{2, p}
$$

is a linear isometry map on $L^{2}\left(\mathcal{D}, C_{2}\right)$.

Note that $L^{2}\left(\mathcal{D}, C_{2}\right)$ is a Hilbert space (compare with Section 3 ).

Proof of Proposition 4.4. For the proof, we need the following theorem of Størmer on structures of Jordan monomorphisms (note that $e$ and $f$ below are orthogonal: ef $=f e=0)$ :

Theorem 4.5 (Størmer [St, Lemma 3.2]). Let $\mathcal{A}, \mathcal{M}$ be von Neumann algebras and $J$ be a normal Jordan $*$-monomorphism from $\mathcal{A}$ into $\mathcal{M}$ such that the von Neumann algebra generated by $J(\mathcal{A})$ equals $\mathcal{M}$. Then there exist two central projections e and $f$ in $\mathcal{M}$ with $e+f=1_{\mathcal{M}}$ such that the map $J_{1}: x \mapsto J(x) e$ is a $*$-homomorphism and that the map $J_{2}: x \mapsto J(x) f$ is an anti-*-homomorphism.

We apply Theorem 4.3 and Theorem 4.5 to our case: $\mathcal{A}=L^{\infty}(\mathcal{D}) \bar{\otimes} \mathbb{B}(\mathfrak{H})$ with the canonical trace $\tau$. Thus for $V$, we obtain $w, J$, and $N$ in the statement of Theorem 4.3 and let $q$ be the projection $J\left(1_{\mathcal{A}}\right) \in \mathcal{A}$. Set $\mathcal{M}$ to be the von Neumann algebra generated by $J(\mathcal{A})$ with putting $1_{\mathcal{M}}=q$, and through Theorem 4.5 we obtain $e, f$ central in $\mathcal{M}$. Take an arbitrary element $x$ in $L_{2}(\mathcal{A}, \tau) \cap \mathcal{A}$, namely, $x$ is an element in $L^{2}\left(\mathcal{D}, C_{2}\right)$ which satisfies ess $\sup _{d \in \mathcal{D}}\|x(d)\|<\infty$. Let $x=u|x|$ be a polar decomposition of $x$ with $u \in \mathcal{A}$ being a unitary. Then

$$
\begin{aligned}
\left(V \circ \tilde{M}_{2, p}\right)(x)=V \cdot\left(u|x|^{2 / p}\right) & =w N J\left(u|x|^{2 / p}\right) e+w N J\left(u|x|^{2 / p}\right) f \\
& =w N J(u) J(|x|)^{2 / p} e+w N J(|x|)^{2 / p} J(u) f .
\end{aligned}
$$


Hence the following is a polar decomposition of $\left(V \circ \tilde{M}_{2, p}\right)(x)$ with $w J(u)$ being a partial isometry:

$$
\begin{aligned}
\left(V \circ \tilde{M}_{2, p}\right)(x) & =(w J(u) e+w J(u) f)\left(N J(|x|)^{2 / p} e+J(u)^{*} N J(|x|)^{2 / p} J(u) f\right) \\
& =(w J(u))\left(N J(|x|)^{2 / p} e+J(u)^{*} N J(|x|)^{2 / p} J(u) f\right) .
\end{aligned}
$$

Here we use the following facts: $J(u)$ is a partial isometry with $J(u)^{*} J(u)=$ $J(u) J(u)^{*}=q ; e, f$ are projections with $e+f=q$ which are central in $\mathcal{M}$; and $N$ is affiliated with $\mathcal{A} \cap \mathcal{M}^{\prime}=\mathcal{A} \cap J(\mathcal{A})^{\prime}$. Therefore, one has the following:

$$
\begin{aligned}
\left(\tilde{M}_{p, 2} \circ V \circ \tilde{M}_{2, p}\right)(x) & =(w J(u))\left(N J(|x|)^{2 / p} e+J(u)^{*} N J(|x|)^{2 / p} J(u) f\right)^{p / 2} \\
& =(w J(u) e+w J(u) f)\left(N^{p / 2} J(|x|) e+J(u)^{*} N^{p / 2} J(|x|) J(u) f\right) \\
& =w N^{p / 2} J(u) J(|x|) e+w N^{p / 2} J(|x|) J(u) f \\
& =w N^{p / 2} J(u|x|) e+w N^{p / 2} J(u|x|) f=w N^{p / 2} J(u|x|) \\
& =w N^{p / 2} J(x) .
\end{aligned}
$$

This means that $\tilde{M}_{p, 2} \circ V \circ \tilde{M}_{2, p}$ is a linear 2-isometry at least from $L_{2}(\mathcal{A}, \tau) \cap \mathcal{A}$ to $L_{2}(\mathcal{A}, \tau)$. Since in our case $L_{2}(\mathcal{A}, \tau) \cap \mathcal{A}$ is $(2-)$ dense in $L_{2}(\mathcal{A}, \tau)$, this map extends to a linear isometry on $L_{2}(\mathcal{A}, \tau)$, as desired.

Theorem 4.2 together with Proposition 4.4 leads us to the following corollary (compare with Section 3):

Corollary 4.6. Let $\mathcal{D}$ be a standard finite measure space and $p \neq 2$ in $(1, \infty)$. Then among locally compact and second countable groups, relative property $(\mathrm{T})$ implies relative property $\left(\mathrm{T}_{L^{p}\left(\mathcal{D}, C_{p}\right)}\right)$.

Proof of Theorem 1.4 for finite index subgroups and Theorem 1.8. First we prove Theorem 1.8. Recall the argument in BFGM, §5] of deducing $\left(\mathrm{F}_{\mathcal{L}^{p}}\right)$ from (strong) relative $\left(\mathrm{T}_{\mathcal{L}^{p}}\right)$ for higher rank algebraic groups (they utilize the generalized HoweMoore property; see [BFGM, §9]). Together with Proposition 1.7 and Corollary 4.6. this argument implies $\left(\mathrm{F}_{\mathcal{C}_{p}}\right)$ and $\left(\mathrm{F}_{L^{p}\left(\mathcal{D}, C_{p}\right)}\right)$ for higher rank algebraic groups. By the $p$-induction process (it is possible; see (2) of Remark 4.1), the latter property implies $\left(\mathrm{F}_{\mathcal{C}_{p}}\right)$ for lattices therein; see [BFGM, §8].

Finally, we end the proof of Theorem 1.4 by dealing with finite index subgroups of universal lattices. Let $G=\mathrm{SL}_{n \geq 4}(A)$ be the universal lattice and $\Gamma \leqslant G$ be a finite index subgroup. Suppose that $\Gamma$ does not have $\left(\mathrm{FF}_{\mathcal{C}_{p}}\right) / \mathrm{T}$. Then there exists an isometric $\Gamma$-representation $\sigma$ on $C_{p}$ and a quasi- $\sigma$-cocycle $b$ such that $b^{\prime}: \Gamma \rightarrow\left(C_{p}\right)_{\sigma(\Gamma)}^{\prime}$ is unbounded. Take a $p$-induction of $b$ (it is possible in view of Remark 4.1 (1)), and get the induced representation $\rho=\operatorname{Ind}_{\Gamma}^{G} \sigma$ and the induced quasi- $\rho$-cocycle $c=\tilde{b}$ in $E=\ell^{p}\left(\mathcal{D}, C_{p}\right)$.

Now observe that Theorem 1.6 and Corollary 4.6 prove that $G$ has $\left(\mathrm{FF}_{E}\right) / \mathrm{T}$. This in particular implies that the map $c^{\prime}: G \rightarrow E_{\rho(G)}^{\prime}$ must be bounded. On the other hand, by the construction of $c$, the restriction $\left.c^{\prime}\right|_{\Gamma}$ can be identified with the quasi- $\sigma^{\prime}$-cocycle $b^{\prime}: \Gamma \rightarrow\left(C_{p}\right)_{\sigma(\Gamma)}^{\prime}$ and hence is unbounded. Since the measure space $\mathcal{D}$ consists of atoms, this forces $c^{\prime}$ to be unbounded. It is a contradiction. Therefore $\Gamma$ must have $\left(\mathrm{FF}_{\mathcal{C}_{p}}\right) / \mathrm{T}$, and also have $\left(\mathrm{F}_{\mathcal{C}_{p}}\right)$ because $\Gamma$ has finite abelianization (for instance, this follows from Theorem 1.11). 
Remark 4.7. Induction of quasi-cocycles from a lattice to a locally compact (topological) group is delicate in general. This is because for a nonatomic finite measure space, the unboundedness of some value does not necessarily imply the unboundedness of the $L^{p}$-norm. However, Burger and Monod have overcome this difficulity by showing that the induced map $H_{\mathrm{b}}^{2}(\Gamma ; B, \sigma) \rightarrow H_{\mathrm{cb}}^{2}\left(G ; L^{p}(\mathcal{D}, B), \operatorname{Ind}_{\Gamma}^{G} \sigma\right)$ is injective (here $H_{\mathrm{cb}}^{\bullet}$ denotes the continuous bounded cohomology). For details, see BM1, BM2].

\section{Concluding Remarks}

Remark 5.1. We will explain an example of a family $\mathcal{B}$ of Banach spaces which satisfies condition $(i)$ but fails to fulfill condition $(i i)$ in Theorem 1.6. This example is employed for establishing property $\left(\mathrm{F}_{[\mathcal{H}]}\right)$ for universal lattices in [Mi], where $[\mathcal{H}]$ denotes the family of Banach spaces $Y$ which is isomorphic to a Hilbert space, namely, on which there exists a Hilbert norm $\|\cdot\|_{\text {Hilb }}$ such that there exists $C \geq 1$ such that $C^{-1}\|\cdot\|_{\text {Hilb }} \leq\|\cdot\|_{Y} \leq C\|\cdot\|_{\text {Hilb }}$ (the infimum of such a $C$ is called the norm ratio). The family $[\mathcal{H}]$ is not stable, but for any $M \geq 1$, the family $[\mathcal{H}]_{M}$ of any $Y$ isomorphic to a Hilbert space with norm ratio $\leq M$ is stable under ultraproducts. Theorem 1.3 in Mi] states that $\mathrm{E}_{2}(A) \ltimes A^{2} \unrhd A^{2}$ has relative property $\left(\mathrm{T}_{[\mathcal{H}]}\right)$, and the family $[\mathcal{H}]_{M}$ satisfies condition $(i)$ of Theorem 1.6. Therefore $\operatorname{SL}_{n \geq 4}(A)$ has property $\left(\mathrm{F}_{[\mathcal{H}]_{M}}\right)$ for any $M$. Thus property $\left(\mathrm{F}_{[\mathcal{H}]}\right)$ for $\mathrm{SL}_{n \geq 4}(A)$ is also verified.

Note that, by considering $\|\cdot\|_{\text {Hilb }}$, one can interpret property $\left(\mathrm{F}_{[\mathcal{H}]}\right)$ as the fixed point property with respect to all affine uniformly bi-Lipschitz actions on Hilbert spaces (similarly, property $\left(\mathrm{T}_{[\mathcal{H}]}\right)$ is interpreted as a property in terms of uniformly bounded linear representations). We note that in BFGM], property $\left(\mathrm{F}_{[\mathcal{H}]}\right)$ and $\left(\mathrm{T}_{[\mathcal{H}]}\right)$ are respectively called property $\left(\bar{F}_{\mathcal{H}}\right)$ and property $\left(\bar{T}_{\mathcal{H}}\right)$.

We also mention that $\mathcal{B}=[\mathcal{H}]$ and $\mathcal{B}=[\mathcal{H}]_{M}$ for sufficiently large $M$ do not satisfy condition (ii) in Theorem 1.6. This is due to an unpublished result of Shalom that $\mathrm{Sp}_{n, 1}$ and the lattices therein fail to have property $\left(\mathrm{T}_{[\mathcal{H}]}\right)$.

Remark 5.2. There is a recent settlement by Olivier related to this remark. See the Added Remark in Section [1

In view of Theorem 4.3. our fixed point theorems (and (FF)/T-type property) can be extended, with some effort, to the cases of noncommutative $L^{p}$-spaces associated with semifinite von Neumann algebras. For uniform continuity of the associated Mazur maps on unit spehres, one can utilize an inequality of Kosaki [Ko, Proposition 7]. Compare with the proof of [Pu, Corollary 5.6].

We mention that even from type III von Neumann algebras (namely, beyond semifinite cases), one can construct the associated noncommutative $L^{p}$-spaces. For details, see [PX]. For classification of linear isometries on a general noncommutative $L^{p}$-space, see [JRS] and [She.

Finally, we warn that the class $\mathcal{C}_{p}$ is not stable under ultraproducts. To have stability, we need to consider noncommutative $L^{p}$-spaces associated with type III von Neumann algebras as well. For details, see [Ra].

Remark 5.3. It is a problem of high interest to determine whether higher rank lattices and universal lattices have property $\left(\mathrm{F}_{\mathcal{B}_{\mathrm{uc}}}\right)$ for $\mathcal{B}_{\mathrm{uc}}$ being the family of all uniformly convex Banach spaces. One of the main motivations for studying this problem is that this relates to uniform (non)embeddability of expander graphs, and 
that relates to the coarse geometric Novikov conjecture $[\mathrm{KY}]$ and (a possible direction to construct a counterexample of the surjectivity-side of) the Baum-Connes conjecture HLS. There is a breakthrough by V. Lafforgue [La1, La2, and his results imply that $\mathrm{SL}_{n \geq 3}(F)$ ( $F$ is a non-Archimedean local field) and cocompact lattices therein have $\left(\mathrm{F}_{\mathcal{B}_{\mathrm{uc}}}\right)$. For Archimedean local field cases or noncocompact lattice cases, it does not seem that any result is known for this problem.

In BFGM], Bader-Furman-Gelander-Monod observed that for higher rank groups and lattices, in order to verify property $\left(\mathrm{F}_{\mathcal{B}_{\mathrm{uc}}}\right)$ it suffices to show that $\mathrm{SL}_{2}(\mathbb{R}) \ltimes \mathbb{R}^{2} \unrhd \mathbb{R}^{2}$ and a symplectic version of this pair have relative property $\left(\mathrm{T}_{\mathcal{B}_{\mathrm{uc}}}\right)$. Thanks to Theorem 1.6. we have an analogue of this observation for universal lattices, namely, the following: if $\mathrm{E}_{2}(A) \ltimes A^{2} \unrhd A^{2}$ has relative property $\left(\mathrm{T}_{\mathcal{B}_{\mathrm{uc}}}\right)$, then $\mathrm{SL}_{n \geq 4}(A)$ has property $\left(\mathrm{F}_{\mathcal{B}_{\text {uc }}}\right)$. The key fact is that the family of uniformly convex Banach spaces with uniform lower bounds for modulus of convexity is stable under ultraproducts [AK], and hence a similar argument to one in Remark 5.1] applies.

\section{ACKNOWLEDGMENTS}

The author thanks his supervisor, Narutaka Ozawa, and Nicolas Monod for comments and conversations. He also thanks Takeshi Katsura and Hiroki Sako, who first raised a question on $p$-Schatten classes to the author. He thanks Bachir Bekka and Baptiste Olivier for fruitful discussions, and the referee for helpful comments. Finally, this work was carried out during a long stay (from February 2010 to January 2011) of the author at EPFL (École Polytechnique Fédérale de Lausanne), sponsored by the Excellent Young Researcher Overseas Visiting Program by the Japan Society for the Promotion of Science. The author is grateful to Professor Nicolas Monod and his secretary, Mrs. Marcia Gouffon, for their warm-hearted hospitality during his stay at EPFL.

\section{REFERENCES}

[AK] A.G. Aksoy and M.A. Khamsi, Nonstandard Methods in Fixed Point Theory. SpringerVerlag, New York, 1990. MR.1066202 (91i:47073)

[Ar] J. Arazy, The isometries of $C_{p}$. Israel J. Math. 22 (1975), 247-256. MR.0512953 $(58: 23760)$

[BFGM] U. Bader, A. Furman, T. Gelander, and N. Monod, Property (T) and rigidity for actions on Banach spaces. Acta Math. 198 (2007), no. 1, 57-105. MR2316269 (2008g:22007)

[BHV] B. Bekka, P. de la Harpe, and A. Valette, Kazhdan's property (T). New Mathematical Monographs, Vol. 11, Cambridge University Press, Cambridge, 2008. MR 2415834 (2009i:22001)

[BL] Y. Benyamini and J. Lindenstrauss, Geometric nonlinear functional analysis. Vol. 1, Amer. Math. Soc., Providence, RI, 2000. MR1727673 (2001b:46001)

[BP] M. Bourdon and H. Pajot, Cohomologie $l_{p}$ et espaces de Besov. J. reine angew. Math. 558 (2003), 85-108. MR1979183 (2004e:20073)

[BM1] M. Burger and N. Monod, Bounded cohomology of lattices in higher rank Lie groups. J. Eur. Math. Soc. 1 (1999), no. 2, 199-235. MR1694584 (2000g:57058a)

[BM2] M. Burger and N. Monod, Continuous bounded cohomology and application to rigidity theory. Geom. Funct. Anal. 12(2) (2002), 219-280. MR1911660(2003d:53065a)

[Co] P. M. Cohn, On the structure of the $G L_{2}$ of a ring. Inst. Hautes Études Sci. Publ. Math. 30 (1966), 5-53. MR0207856 (34:7670)

[FP] T. Figiel and G. Pisier, Séries aléatories dans les espaces uniformément convexes ou uniformément lisses. C. R. Acad. Sci. Paris Sér. A 279 (1974), 611-614. MR0358295 (50:10761) 
[FJ] R. Fleming and J. Jamison, Isometries on Banach spaces: function spaces. Chapman \& Hall/CRC, Boca Raton, 2003. MR 1957004 (2004j:46030)

[Gr] M. Gromov, Random walks in random groups. Geom. Funct. Anal. 13 (2003), 73-148. MR1978492(2004j:20088a)

[He] S. Heinrich, Ultraproduct in Banach space theory. J. reine angew. Math. 313 (1980), 72-104. MR552464 (82b:46013)

[HLS] N. Higson, V. Lafforgue, and G. Skandalis, Counterexamples to the Baum-Connes conjecture. Geom. Funct. Anal. 12 (2002), 330-354. MR1911663 (2003g:19007)

[JRS] M. Junge, Z.-J. Ruan, and D. Sherman, A classification for 2-isometries of noncommutative $L_{p}$-spaces. Isr. J. Math. 150 (2005), 285-314. MR2255812 (2008d:46084)

[KY] G. Kasparov and G. Yu, The coarse geometric Novikov conjecture and uniform convexity. Adv. Math. 206 (2006), no. 1, 1-56. MR2261750 (2007g:58025)

[Ka] D. A. Kazhdan, Connection of the dual space of a group with the structure of its closed subgroups. Funct. Anal. Appl. 1 (1967), 63-65. MR0209390 (35:288)

[Ko] H. Kosaki, On the convexity of the map $\phi \rightarrow|\phi|$ from the predual of a $W^{*}$-algebra. J. Funct. Anal. 59 (1984), 123-131. MR763779 (86c:46072)

[La1] V. Lafforgue, Un renforcement de la propriété (T). Duke Math. J. 143 (2008), 559-602. MR2423763(2009f:22004)

[La2] V. Lafforgue, Propriété (T) renforcée banachique et transformation de Fourier rapide. J. Topol. Anal. 1 (2009), no. 3, 191-206. MR2574023 (2010m:22004)

[LMR] A. Lubotzky, S. Mozes, and M. S. Raghunathan, The word and Riemannian metrics on lattices of semisimple groups. Inst. Hautes Études Sci. Publ. Math. 91 (2000), 5-53. MR 1828742(2002e:22011)

[Mi] M. Mimura, Fixed-point properties and second bounded cohomology of universal lattices on Banach spaces. J. reine angew. Math. 653 (2011), 115-134. MR2794627

[Mo] N. Monod, Continuous bounded cohomology of locally compact groups. Springer Lecture Notes in Mathematics, 1758, 2001. MR1840942 (2002h:46121)

[MMS] I. Mineyev, N. Monod, and Y. Shalom, Ideal bicombings for hyperbolic groups and applications. Topology 43 (2004), no. 6, 1319-1344. MR2081428(2005i:20067)

[Na] A. Navas, Reduction of cocycles and groups of diffeomorphisms of the circle. Bull. Belg. Math. Soc. Simon Stevin 13 (2006), no. 2, 193-205. MR2259900 (2007g:37030)

[Ol] B. Olivier, Kazhdan's property $(T)$ with respect to non-commutative $L_{p}$-spaces. Preprint, arXiv:1107.1394, to appear in Proc. Amer. Math. Soc.

[Pa] P. Pansu, Cohomologie $L^{p}$ : invariance sous quasiisométrie, preprint, 1995.

[PX] G. Pisier and Q. Xu, Non-commutative $L^{p}$-spaces. In Handbook of the geometry of Banach spaces, Vol. 2, 1459-1517. North-Holland, Amsterdam, 2003. MR.1999201 (2004i:46095)

[Pu] M. Puschnigg, Finitely summable Fredholm modules over higher rank groups and lattices. J. K-Theory 8 (2011), no. 2, 223-239. MR2842930

[Ra] Y. Raynaud, On ultrapowers of non commutative $L_{p}$ spaces. J. Operator Theory 48 (2002), no. 1, 41-68. MR1926043 (2003i:46069)

[Sha1] Y. Shalom, Bounded generation and Kazhdan's property (T). Inst. Hautes Études Sci. Publ. Math. 90 (1999), 145-168. MR.1813225 (2001m:22030)

[Sha2] Y. Shalom, Rigidity of commensurators and irreducible lattices. Invent. Math. 141(1) (2000), 1-54. MR 1767270 (2001k:22022)

[Sha3] Y. Shalom, Rigidity, unitary representations of semisimple groups, and fundamental groups of manifolds with rank one transformation group. Ann. of Math. (2) 152 (2000), 113-182. MR 1792293 (2001m:22022)

[Sha4] Y. Shalom, The algebraization of Kazhdan's property (T). International Congress of Mathematicians. Vol. II, Eur. Math. Soc., Zürich (2006), 1283-1310. MR2275645 (2008a:22003)

[She] D. Sherman, Noncommutative $L^{p}$-structure encodes exactly Jordan structure. J. Funct. Anal. 221 (2005), 150-166. MR2124900 (2006c:46051)

[St] E. Størmer, On the Jordan structure of $C^{*}$-algebras. Trans. Amer. Math. Soc. 120 (1965), 438-447. MR0185463 (32:2930)

[Su] A. A. Suslin, On the structure of the special linear group over polynomial rings. Math. USSR Izv. 11 (1977), 221-238. MR0472792 (57:12482) 
[Va] L. Vaserstein, Bounded reduction of invertible matrices over polynomial ring by addition operators, preprint, 2007.

[Ye] F. J. Yeadon, Isometries of non-commutative $L^{p}$ spaces. Math. Proc. Camb. Phil. Soc. 90 (1981), 41-50. MR611284 (82g:46108)

[Yu] G. Yu, Hyperbolic groups admit proper affine isometric actions on $\ell^{p}$-spaces. Geom. Funct. Anal. 15 (2005), 1144-1151. MR2221161 (2007f:20075)

Graduate School of Mathematical Sciences, University of Tokyo, Komaba, Tokyo, 153-8914, Japan - And - École Polytechnique Fédérale de Lausanne, SB-IMB-EGG, StaTion 8, BÂtiment MA, Lausanne, VAud, CH-1015, Switzerland

E-mail address: mimurac@ms.u-tokyo.ac.jp

Current address: Graduate School of Mathematical Sciences, University of Tokyo, Komaba, Tokyo, 153-8914, Japan - and - Institut de Mathématiques, Faculté des Sciences, Université de Neuchâtel, Rue Emile-Argand 11, CH-2000 Neuchâtel, Switzerland 